\title{
Evaluación de los Métodos de Localización del Punto Pronasal para la Reconstrucción Facial Forense
}

\author{
Evaluation of the Pronasal Point Prediction Methods for Forensic Facial Reconstruction
}

\author{
Bernarda López; Juan Schilling \& Iván Suazo Galdames
}

\begin{abstract}
LÓPEZ, B.; SCHILLING, J.\& SUAZO, G. I. Evaluación de los métodos de localización del punto pronasal para la reconstrucción facial forense. Int. J. Morphol., 28(4):1181-1188, 2010.

RESUMEN: La reconstrucción facial por escultura forense es un procedimiento que se utiliza en identificación Humana. Consiste en la colocación de un material modelable sobre el esqueleto óseo, respetando los grosores tisulares y la anatomía facial. Una de las principales dificultades de la técnica es la reproducción de estructuras de posición variable como los ojos, las orejas y la nariz. Se ha descrito que es posible reconstruir la nariz de manera confiable si se cuenta con el punto pronasal, es por esto que se han desarrollado diversas técnicas para la ubicación de este punto a partir de osamentas craneales. El propósito de este estudio es evaluar mediante radiografías laterales la exactitud de 4 métodos ampliamente utilizados para la ubicación del punto pronasal a partir de cráneos. Se utilizaron 34 radiografías tomadas en norma lateral con perfil blando visible, sobre los cuales se realizaron los trazados de perfil blando y duro (perfil de control). Se evaluaron los métodos de Gerásimov, krogman, Prokopec \& Ubelaker y de George, determinándose las diferencias existentes en un eje de coordenadas (X, Y) con respecto al gold estándar (punto pronasal determinado en el perfil blando). Todos los métodos presentaron bajos niveles de exactitud, subestimando la proyección anterior de la nariz. De acuerdo a las medias el método de Prokopec \& Ubelaker fue el más cercano al gold standard, con medias de 5,6 (DS 3,66) en X y 1,7 (DS 3,3) en Y, y mayores niveles de exactitud, los otros métodos evaluados fueron menos exactos y presentaron una alta dispersión. El uso de métodos de determinación del punto pronasal confiables y aplicables a nuestra población es fundamental para la correcta reconstrucción de la nariz.
\end{abstract}

PALABRAS CLAVE: Punto pronasal; reconstrucción forense; identificación Humana.

\section{INTRODUCCIÓN}

La reconstrucción facial por escultura forense es un procedimiento en el cual son adicionados materiales moldeables sobre un sustrato óseo, un cráneo completo o previamente reconstituido. Este método permite aproximarnos a la fisionomía de los tejidos blandos, lo que facilitará la identificación y es útil tanto en ciencias forenses como en arqueología y paleobiología (Gerasimov, 1971; Gatliff, 1984; Krogman \& Iscan, 1986; Prag y Neave, 1997).

Otros métodos permiten una reconstrucción sobre un plano bidimensional, como imágenes fotográficas y radiográficas. Cualquiera sea el método empleado estos deben conjugar el reproducir adecuadamente las estructuras anatómicas subyacentes, junto con respetar las características del tejido blando facial, la presencia de dimorfismo sexual y su comportamiento población específico (Suazo et al., 2008a; Suazo et al., 2008c; Suazo et al., 2008d; Suazo et al., 2009). En la literatura destacan los métodos descritos por Gerasimov, que requiere conocimiento substancial de la anatomía facial para predecir la morfología individual del músculo, y el método de Krogman \& Iscan, que utiliza principalmente medidas de la profundidad del tejido fino en numerosos puntos óseos del cráneo. Estas dos técnicas se pueden combinar para generar métodos más confiables en la reconstrucción anatómica de la forma de la cara, y los datos de la profundidad de tejido para dirigir al clínico.

El hueso es un soporte rígido sobre el cual se anclan los tejidos blandos subyace en las técnicas propuestas para la reconstrucción facial, sin embargo, los tejidos duros y blandos se modifican conjuntamente en el transcurso de la vida, principalmente debido a la función. Por lo tanto, la forma del cráneo es creada por las fuerzas internas y externas ejercidas in-vivo sobre él por el tejido blando y por el 
desarrollo evolutivo del tejido blando. Por lo tanto, la relación del tejido blando con el duro es parcialmente recíproca (Enlow \& Hans, 1996; Larsen, 2001).

No obstante la importancia de considerar al hueso como un determinante activo de la forma, los autores coinciden en que un cráneo intacto o bien reconstruido es un buen soporte para el modelado de las partes blandas. El problema surge en ciertas estructuras que estando contenidas en cavidades óseas o tomando soporte en hueso varían considerablemente en los sujetos vivos, como los bulbos oculares, el pelo, la nariz y las orejas. La reconstrucción de estas estructuras puede ser clave para conseguir la identificación por parte de familiares en casos de interés forense.

En la actualidad existen variados métodos que buscan predecir la forma externa de la nariz a partir de osamentas, aunque algunos autores creen que es imposible predecir exactamente la forma de la nariz tomando como base la abertura piriforme (Virchow, 1912, 1924; George, 1993), o no hay correlación entre la forma de la cavidad nasal y la nariz blanda (Suk, 1935). No obstante lo anterior, diversos autores han reportado que es posible determinar la localización del punto pronasal a partir de cráneos y que este punto es una buena orientación para la reconstrucción de la nariz, ya que le aporta un punto de referencia al dorso nasal y a las regiones subnasal y alar (Gerasimov 1971; Krogman e Iscan; Prag y Neave ; Wilkinson, 2004).

Gerasimov desarrolló un método para la predicción del punto pronasal, basado en la inclinación de la espina nasal anterior y los huesos nasales. Krogman (1962) desarrolló un método para la predicción del punto pronasal, a partir de las dimensiones de la espina nasal anterior, él reportó que la proyección blanda de la nariz era igual a tres veces la longitud de la espina nasal anterior. Posteriormente Krogman \& Iscan (1986) adicionaron el grosor promedio del tejido blando reportado para la región del pronasal. Un tercer método fue sugerido por Macho (1986), concebido después de la hipótesis de Goldhamer (1926) de la cefalometría multivariada del cráneo que correlacionada con medidas craneométricas externas resultó de poca utilidad práctica. George (1987) diseñó un método basado en dimensiones lineales de la región craneofacial, las que permitían establecer una proporción en base a la cual se determinaba la localización del pronasal. Finalmente Prokopec \& Ubelaker (2002) diseñaron un método basado en la relación entre la línea nasion-prostion y los márgenes de la apertura piriforme. Estos diferentes métodos han sido evaluados con resultados diversos Stephan et al. (2003), siendo necesaria su validación en diferentes poblaciones, esto debido a que su desarrollo ha sido sobre población norteamericana y europea. No existen estudios de la validez de los métodos más utilizados en población sudamericana.
Con estos antecedentes, el propósito de este estudio es evaluar mediante radiografías laterales la exactitud de 4 métodos ampliamente utilizados para la ubicación del punto pronasal a partir de cráneos.

\section{MATERIAL Y MÉTODO}

Se utilizaron 34 radiografías de cráneos tomadas en norma lateral estricta (14 hombres y 20 mujeres) con una media de edad de 24,3 años (DS 3,3). Las radiografías fueron seleccionadas de la Base de Datos del Servicio de Radiología Maxilofacial del Centro de Clínicas de la Universidad de Talca. Se incluyeron sólo radiografías de pacientes clase I esqueletal, sin alteraciones morfológicas evidentes.

Procedimiento:Sobre cada imagen se superpuso un papel vegetal, en el cual se trazó el perfil blando y duro como control y el perfil duro en 4 ocasiones más, para la aplicación de 4 técnicas de predicción del punto pronasal frecuentemente utilizadas en reconstrucción facial. Sobre todos los trazados se agregaron los puntos porion y orbitario, con los que se trazó el plano de Frankfurt.

Sobre los trazados de perfil duro se aplicaron las técnicas como se describe a continuación:

1. Método Gerásimov: Este método descrito en 1955 y publicado posteriormente indica que el punto pronasal está determinado por la intersección de dos líneas, la primera de ellas se traza de posterior a anterior siguiendo la dirección general de la espina nasal anterior (ENA). Para trazar la segunda se deben dividir los huesos nasales en tercios, luego, se traza una tangente que siga la dirección del tercio distal de los huesos nasales hacia anterior, la intersección entre ambas líneas muestra la ubicación de pronasal (Fig. 1).

2. Método de Krogman: Se proyecta una línea siguiendo la dirección de la espina nasal anterior (ENA), luego se mide la distancia desde la unión del vómer con el maxilar a la ENA, esta distancia es triplicada desde la ENA hacia anterior sobre la línea ya trazada, obteniéndose el punto pronasal. (según la técnica original de Krogman el pronasal sería ubicado sumando tres veces el largo de la ENA.) Si la unión del vómer con el maxilar no es visible, se ocupa la distancia entre la espina nasal anterior y el borde lateral de la abertura piriforme en el perfil (Stephant et al.) (Fig. 2).

3. Método de Prokopec \& Ubelaker: Se traza un plano desde el punto nasion al punto prostion (línea A), luego se dibuja una línea paralela a la anterior desde rhinion hacia 


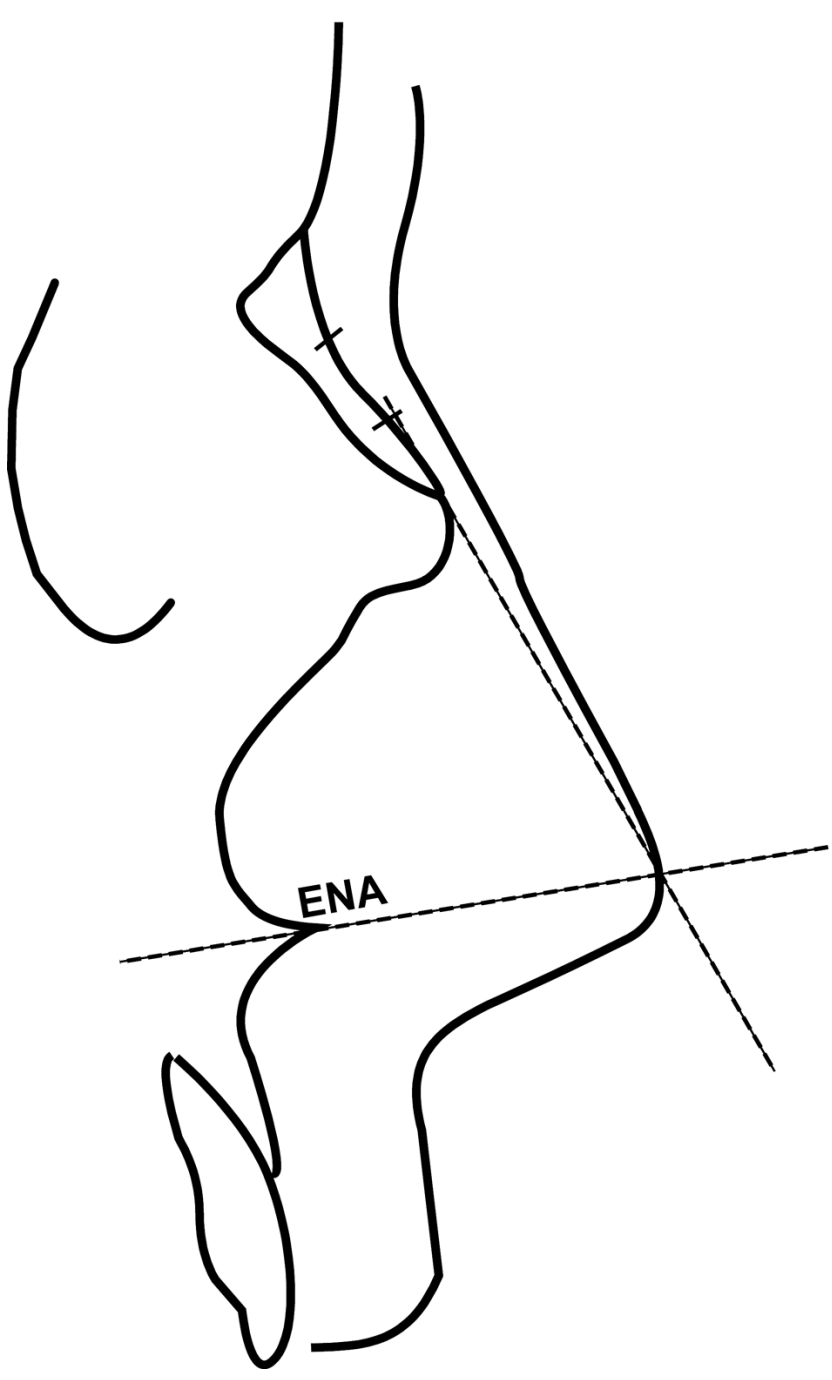

Fig. 1. Técnica de Gerásimov o de las dos tangentes, se muestra la línea que sigue la dirección general de la ENA y la segunda que sigue el tercio distal de los huesos nasales, la intersección de ambas líneas determina el punto pronasal.

inferior abarcando completamente la abertura nasal. (Línea B), esta línea se divide en siete partes iguales (puntos $\mathrm{X}$ ). La distancia desde cada punto $\mathrm{X}$ a la abertura nasal se mide $\mathrm{y}$ proyecta como imagen en espejo hacia anterior, agregándose dos milímetros que corresponden al grosor del tejido blando (puntos $\mathrm{P}$ ), el punto $\mathrm{P}$ más anterior correspondería al punto pronasal (Fig. 3).

4. Método de George : Se debe trazar el plano de Frankfurt, posteriormente se dibuja y mide la distancia desde nasion al punto A (punto de mayor profundidad de la superficie curva existente entre la ENA y el prostion) este trazo fue llamado

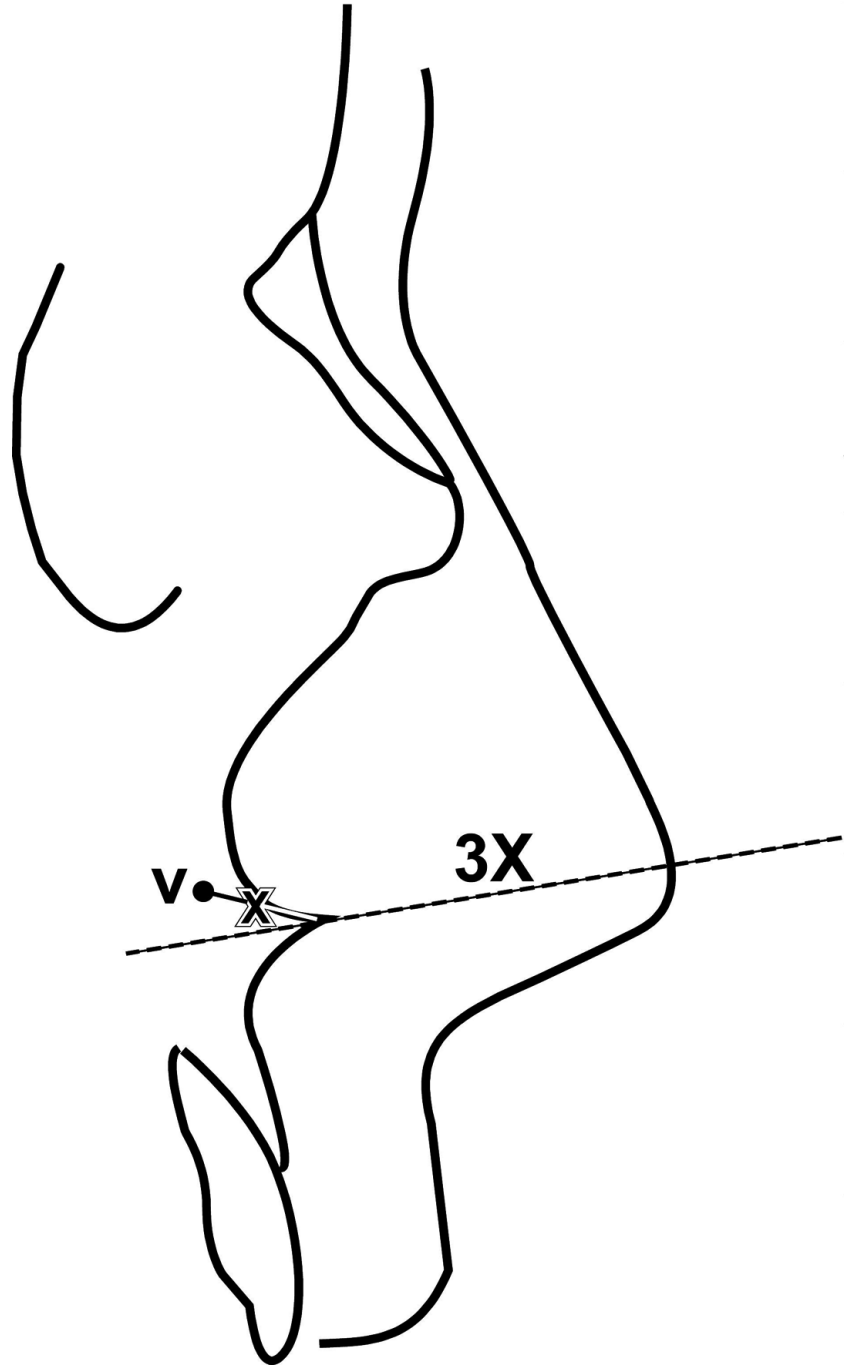

Fig. 2. Técnica de Krogman. La distancia desde el punto V (unión del vómer con el maxilar) al vértice de la espina nasal anterior se ha marcado como $\mathrm{X}$, esta distancia es triplicada hacia anterior desde el vértice de la espina nasal anterior sobre la línea que marca la dirección general de la ENA. El fin de esta distancia determina el punto pronasal.

Línea L. Luego se ubica la vertiente inferior de la ENA marcando el punto medio de dicha vertiente (punto AA), posteriormente, a partir del punto AA, se traza una línea paralela al plano de Frankfurt, esta es llamada línea F. En una última etapa se determina la longitud de la línea $\mathrm{L}$ (Nasion-punto A) y se identifica el punto de intersección con la línea F. A partir de este punto la línea $F$ es proyectada hacia anterior, en una extensión diferente para hombres y mujeres, terminando en el punto Pronasal. La extensión de la línea $\mathrm{F}$ desde el punto de intersección será $60.5 \%$ de la longitud de la línea L para hombres y 56\% de la longitud de la línea L para mujeres (Fig. 4). 


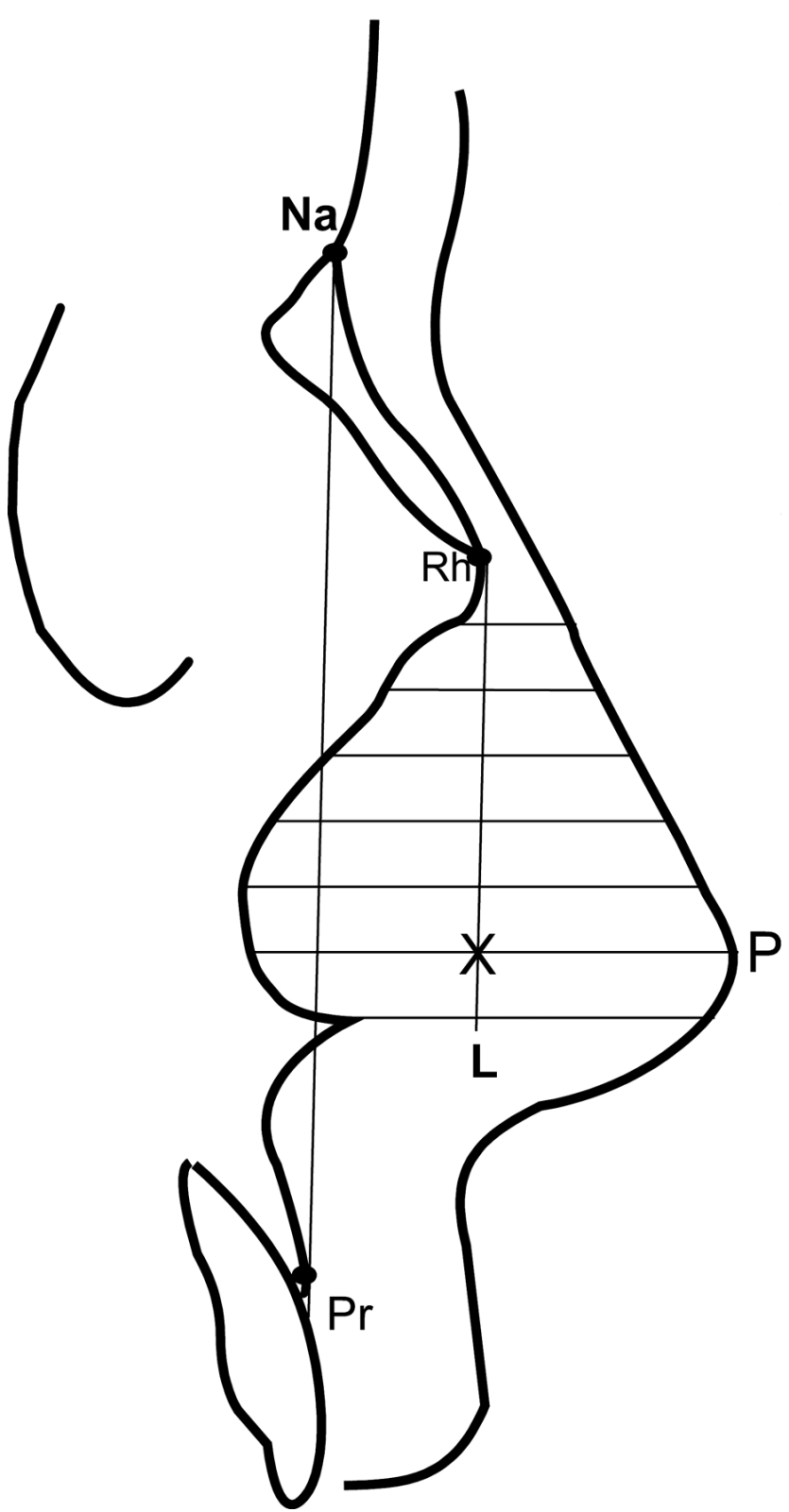

Figura 3: Técnica de Prokopec \& Uberlaker. Se muestra la línea Nasion - Prostion y la línea paralela a ésta que va desde el Rhinion hacia inferior abarcando completamente la abertura nasal (Línea B) la cual se divide en 7 partes iguales (que llamaremos puntos $\mathrm{X})$. Luego se mide la distancia desde la abertura nasal a la línea B en los puntos marcados (X), esta distancia se agrega hacia anterior como imagen en espejo sumando además $2 \mathrm{~mm}$ (correspondiente al grosor de tejido blando). A este punto lo llamaremos puntos $\mathrm{P}$, siendo el punto $\mathrm{P}$ más anterior el punto pronasal.

Determinación del Gold Standard: Sobre el trazado del perfil blando se marcó el punto pronasal definido como: el punto más anterior en la nariz cuando la cabeza se alinea en el plano horizontal de Frankfurt. Esta etapa fue realizada por un investigador diferente a quien diseñó el trazado y aplicó las técnicas.

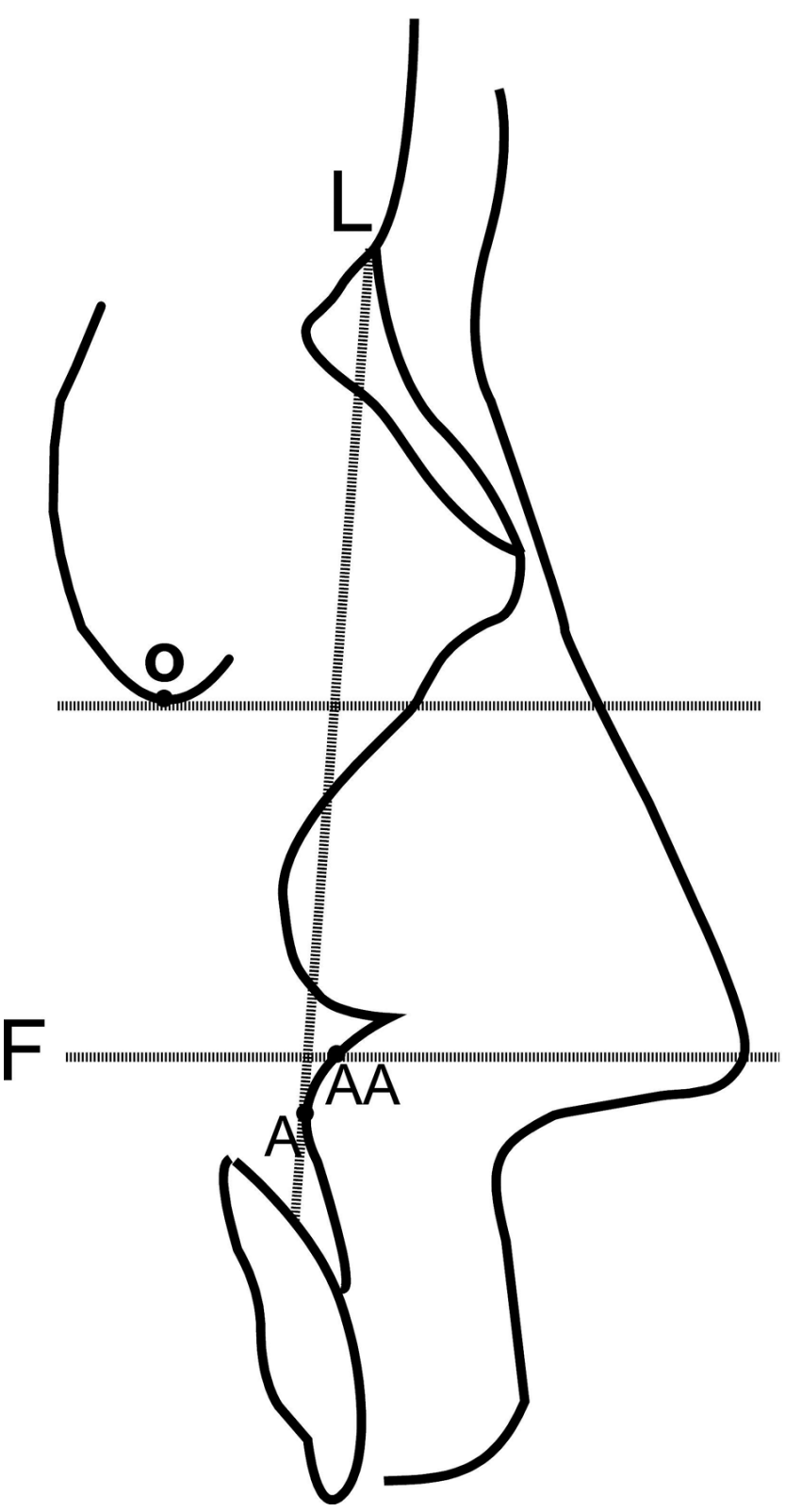

Fig. 4. Técnica de George: Se observa la línea Nasion - punto A (línea de mayor profundidad del maxilar entre ENA y Prostion) que llamaremos Línea L. En AA se determina la zona media de la vertiente inferior de la ENA. Se observa trazado el plano de Frankfurt y una línea paralela a este plano que pasa por el punto AA. Se mide la distancia desde el punto Nasion al punto A y se agrega hacia anterior sobre la Línea F desde la intersección de esta con la línea L, en una proporción de $60.5 \%$ de dicha línea en los hombres y $56 \%$ para las mujeres, el fin de esta línea determina el punto pronasal.

Evaluación de los métodos: En una última etapa se colocó el perfil de control de los individuos bajo cada una de las técnicas (una cada vez) sobre un papel milimetrado que contenía un eje de coordenadas X e Y. Se utilizó el plano de Frankfurt ya trazado el que fue estrictamente ubicado en una 
de las líneas horizontales del papel. El punto pronasal de cada perfil blando se posicionó en el centro del eje de coordenadas para estandarizar los trazados.

Posteriormente se midieron las distancias, en el eje de coordenadas $\mathrm{X}$ e Y, desde los puntos pronasales encontrados al aplicar cada una de los métodos con respecto al punto pronasal determinado sobre el perfil blando (gold standard). Con los datos obtenidos se calcularon los estadísticos descriptivos de las distancias en el eje X e Y.

Determinación de la exactitud del los métodos: Se consideró que el resultado de la predicción del punto pronasal era positivo cuando la distancia con respecto al gold standard fue igual o menor que $5 \mathrm{~mm}$ en cualquier sentido del espacio analizado en nuestro eje de coordenadas, a distancias superiores a $5 \mathrm{~mm}$ se consideró que el resultado de la predicción era negativo. Con esta clasificación se calculó la exactitud del método en el plano horizontal, vertical e integrado en los dos ejes.

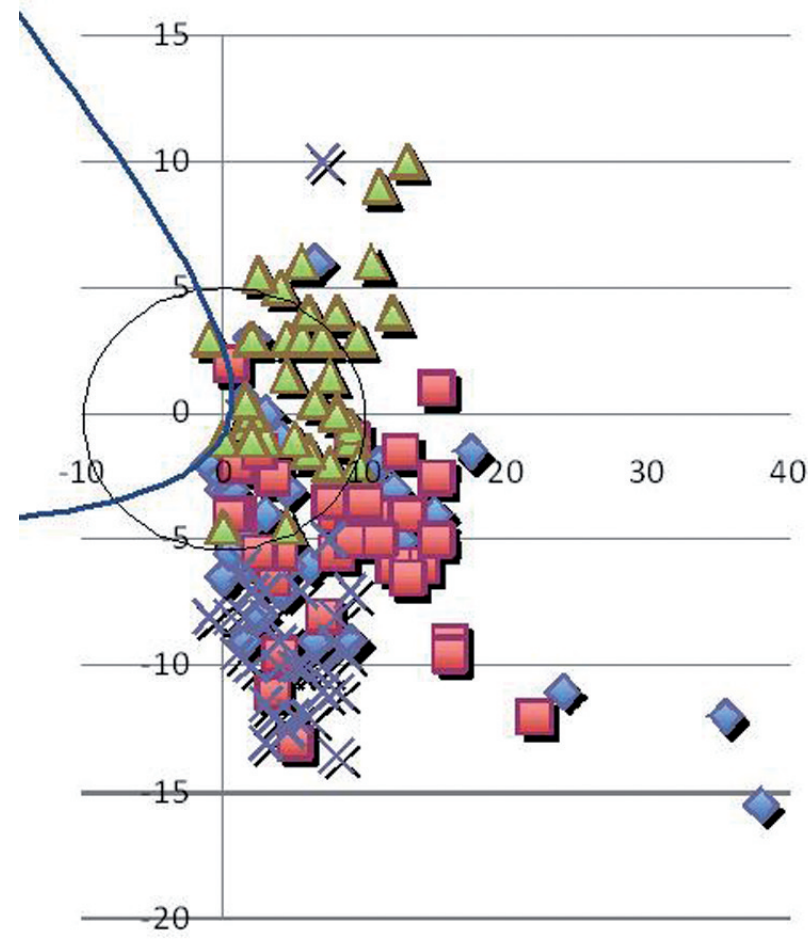

\section{RESULTADOS}

De acuerdo a las medias el método de Prokopec \& Ubelaker fue el más cercano al gold standard, con medias de 5,6 (DS 3,66) en X y 1,7 (DS 3,3) en Y, los otros métodos evaluados fueron menos exactos y presentaron una alta dispersión. El detalle de estos resultados se encuentra en la Tabla I.

La Figura 5 grafica la dispersión de los puntos en los cuales se localizó el punto pronasal al aplicar los cuatro métodos evaluados

Al considerar la exactitud de manera integrada en los dos ejes, los mayores niveles de exactitud se obtuvieron al aplicar el método de Prokopec \& Ubelaker (50-66\%), seguido del método de Gerásimov (21-40\%), luego el de Krogman (14,2-20\%), en todos los casos este indicador fue mayor en el grupo de mujeres. El método de George presen-

$\triangle$ Método Técnica 1
$\square$ Método Técnica 2
$\triangle$ Método Técnica 3
XMétodo Técnica 4

Fig. 5. Dispersión de los puntos en los cuales se localizó el punto pronasal al aplicar los cuatro métodos evaluados. El círculo delimita el área dentro de la cual se consideró positiva la predicción del punto pronasal. La línea azul indica la punta de la nariz. Método Técnica 1. de Gerásimov; Método Técnica 2. de Krogman; Método Técnica 3. de Prokopec \& Ubelaker; Método Técnica 4. de George.

Tabla I. Distancias medias del punto pronasal, de acuerdo a la predicción realizada en los cuatro métodos analizados, con respecto al gold standard.

\begin{tabular}{ccccccccc}
\hline \multirow{2}{*}{$\begin{array}{c}\text { Distancia con } \\
\text { respecto al } \\
\text { Gold Standard }\end{array}$} & \multicolumn{2}{c}{ Método Gerásimov } & \multicolumn{2}{c}{ Método de Krogman } & \multicolumn{2}{c}{$\begin{array}{c}\text { Método de Prokopec } \\
\text { \& Ubelaker }\end{array}$} & \multicolumn{2}{c}{ Método de George } \\
\cline { 2 - 10 } & Eje X & Eje Y & Eje X & Eje Y & Eje X & Eje Y & Eje X & Eje Y \\
\hline Media (mm) & 7,5 & $-4,7$ & 8,6 & $-5,2$ & 5,6 & 1,7 & 4,9 & $-9,0$ \\
DS & 9,2 & 4,1 & 5,4 & 3,4 & 3,6 & 3,3 & 2,6 & 3,9 \\
\hline
\end{tabular}


tó $0 \%$ de exactitud al evaluar el resultado del método con los ejes $\mathrm{X}$ e Y integrados.

El detalle de los valores de exactitud en los ejes X e Y y de manera integrada se encuentran en la Tabla II para el método de Gerásimov; Tabla III para el método de Krogman; Tabla IV para el método de Prokopec \& Ubelaker y Tabla V para el método de George.

Tabla II. Exactitud del método de Gerásimov en los ejes X e Y y de manera integrada aplicado en 34 radiografías de cráneo.

\begin{tabular}{ccccccc}
\hline & \multicolumn{5}{c}{ Método Gerásimov } \\
\cline { 2 - 7 } & \multicolumn{2}{c}{ Eje X } & \multicolumn{2}{c}{ Eje Y } & \multicolumn{2}{c}{ Integrado $(\mathrm{X}, \mathrm{Y})$} \\
\cline { 2 - 7 } Positivo & Hombre & Mujer & Hombre & Mujer & Hombre & Mujer \\
\cline { 2 - 7 } Negativo & 7 & 13 & 6 & 12 & 3 & 8 \\
Exactitud & 7 & 7 & 8 & 8 & 11 & 12 \\
& $50 \%$ & $65 \%$ & $42,8 \%$ & $60 \%$ & $21 \%$ & $40 \%$ \\
\hline
\end{tabular}

Tabla III. Exactitud del método de Krogman en los ejes X e Y y de manera integrada aplicado en 34 radiografías de cráneo.

\begin{tabular}{lcccccc}
\hline & \multicolumn{5}{c}{ Método de Krogman } \\
\cline { 2 - 7 } & \multicolumn{2}{c}{ Eje X } & \multicolumn{2}{c}{ Eje Y } & \multicolumn{2}{c}{ Integrado (X,Y) } \\
\cline { 2 - 7 } Positivo & Hombre & Mujer & Hombre & Mujer & Hombre & Mujer \\
\cline { 2 - 7 } Negativo & 4 & 8 & 8 & 11 & 2 & 4 \\
Exactitud & 10 & 12 & 6 & 9 & 12 & 16 \\
& $28,5 \%$ & $40 \%$ & $57,1 \%$ & $55 \%$ & $14,2 \%$ & $20 \%$ \\
\hline
\end{tabular}

Tabla IV. Exactitud del método de Prokopec \& Ubelaker en los ejes X e Y y de manera integrada aplicado en 34 radiografías de cráneo.

\begin{tabular}{lcccccc}
\hline & \multicolumn{5}{c}{ Método de Prokopec \& Ubelaker } \\
\cline { 2 - 7 } & \multicolumn{2}{c}{ Eje X } & \multicolumn{2}{c}{ Eje Y } & \multicolumn{2}{c}{$\begin{array}{c}\text { Integrado } \\
(\mathrm{X}, \mathrm{Y})\end{array}$} \\
\cline { 2 - 7 } Positivo & 7 & 9 & 14 & 15 & 7 & 8 \\
Hombre & Mujer & Hombre & Mujer & Hombre & Mujer \\
Negativo & 7 & 11 & 0 & 5 & 7 & 12 \\
Exactitud & $50 \%$ & $45 \%$ & $100 \%$ & $75 \%$ & $50 \%$ & $66,6 \%$ \\
\hline
\end{tabular}

Tabla V. Exactitud del método de George en los ejes X e Y y de manera integrada aplicado en 34 radiografías de cráneo.

\begin{tabular}{ccccccc}
\hline & \multicolumn{5}{c}{ Método de George } \\
\cline { 2 - 7 } & \multicolumn{2}{c}{ Eje X } & \multicolumn{2}{c}{ Eje Y } & \multicolumn{2}{c}{ Integrado (X,Y) } \\
\cline { 2 - 7 } Positivo & Hombre & Mujer & Hombre & Mujer & Hombre & Mujer \\
\cline { 2 - 7 } Negativo & 4 & 13 & 0 & 1 & 0 & 0 \\
Exactitud & 10 & 7 & 14 & 19 & 14 & 20 \\
& $28,5 \%$ & $65 \%$ & $0 \%$ & $5 \%$ & $0 \%$ & $0 \%$ \\
\hline
\end{tabular}

\section{DISCUSIÓN}

La localización del punto pronasal es fundamental para la reconstrucción de la nariz necesaria para realizar una aproximación a la forma facial a partir de cráneos. En el presente estudio evaluamos 4 métodos ampliamente utilizados para predecir la ubicación del punto pronasal, a partir de radiografías laterales de cráneo, ya que en estas proyecciones es posible determinar con exactitud el punto pronasal sobre el perfil blando que aparece en las imágenes, de manera de que establecimos un gold standard a partir del cual evaluamos los 4 métodos. La delimitación del perfil óseo en las radiografías permitió la aplicación de los métodos, sin embargo, fueron frecuentes las situaciones en las que la delimitación de las estructuras necesarias para aplicar la técnica fue difícil (ejemplo la unión vomeromaxilar para el método de Krogman), lo que sugiere limitaciones al uso de radiografías para la evaluación de estos métodos y variaciones entre los diferentes estudios. Estas dificultades también fueron reportadas por Stephan et al.

$\mathrm{Al}$ analizar los métodos en su conjunto vemos que la mayor parte de los datos se encuentran en el sector positivo del eje $X$, lo que se interpreta como una sobreestimación de la proyección anterior del punto pronasal. Diferente a lo que ocurre en el eje Y, donde encontramos sólo al método de Prokopec \& Ubelaker en el sector positivo del eje, mientras que los otros métodos se encontraron en el sector negativo. Debido a esto el método de Prokopec \& Ubelaker ubicó el punto pronasal desplazado hacia superior, mientras que los otros métodos lo ubicaron orientado hacia inferior en este eje. Llama la atención la gran dispersión que presentan los datos, lo que se expresa en bajos niveles de exactitud general de los métodos analizados.

El método de Gerasimov presentó niveles adecuados de exactitud (50-65\%) en el plano anteroposterior (eje $\mathrm{X}$ ), probablemente debido a que el elemento que determina esta orientación en este método es 
la inclinación de la espina nasal anterior, aún cuando tiende a sobreestimación la proyección anterior del punto pronasal, lo que es más notorio en las muestras de hombres. En plano vertical (eje Y) este método es menos exacto y desplaza el punto pronasal hacia inferior. Estos resultados difieren de lo reportado por Stephan et al. y por Stephan \& Henneberg (2001) que lo consideran un método muy exacto para la predicción del punto pronasal.

El método de Krogman tiene niveles más bajos de exactitud, este método sobreestimar la proyección de la punta de la nariz en 8,6 mm promedio, estos valores son diferentes a los que reporta Stephan et al. quienes encontraron que el método subestimaba la proyección nasal en 1.9 milímetros en varones y 4.1 milímetros en mujeres. En nuestro estudio la principal dificultad fue la localización de la unión vomeromaxilar, lo que sugiere que los bajos niveles de exactitud de este método al evaluarlo usando radiografías pueden revertirse al realizar una observación directa en cráneos, debemos sumar además que la técnica que se analiza es la de Kogman sin Iscan, es decir no se agrega el grosor promedio de tejidos blandos para pronasal.

El método de Prokopec \& Ubelaker fue el que presentó mejores niveles de exactitud, especialmente en el plano vertical (eje Y) tanto en hombres como en mujeres. Este método se basa en una proyección en espejo del margen de la apertura piriforme, la principal ventaja está en la fácil realización por el uso de referencias fácilmente identificables. Este método se basa en una relación proporcional entre las estructuras duras que sustentan la nariz y las partes blandas que le dan la forma, estas relaciones de pro- porcionalidad han sido descritas por diversos autores y son una característica de la arquitectura craneofacial (Trujillo et al., 2007; Suazo et al., 2008b; Troncoso et al., 2008).

El método de George presentó los niveles más bajos de exactitud en la predicción del punto pronasal, este método ubica la punta de la nariz a partir de una serie de indicadores indirectos: una línea paralela al plano de Frankfurt y una proporción variable en hombres y mujeres de la longitud nasion - punto A, es probable que por ello el método sea muy dependiente del operador y que sus resultados presenten una validez limitada en otros contextos poblacionales, este es el método que utiliza menos puntos de referencia anatómicos estables, por lo que su utilización no parece recomendable en nuestra población. Además de que la técnica no da una definición de localización en plano vertical, (Stephan et al.) por lo que tiende a la subestimación del punto pronasal en este eje.

En conclusión, nuestro estudio evalúo cuatro métodos ampliamente utilizados para la predicción del punto pronasal, diseñados para ser aplicados en cráneos. La evaluación radiográfica de los métodos presentó limitaciones relacionadas con la delimitación de algunas estructuras, no obstante los métodos pudieron aplicarse presentando niveles bajos de exactitud general, frecuentemente sobreestimando la proyección anterior de la nariz. Nuestros resultados sugieren que estos métodos presentan baja utilidad para ser aplicados en nuestra población, por lo que resulta necesario introducir modificaciones y/o nuevos métodos que permitan la predicción de la ubicación del pronasal a partir de cráneos de origen local.

LÓPEZ, B.; SCHILLING, J.\& SUAZO, G. I. Evaluation of the pronasal point prediction methods for forensic facial reconstruction. Int. J. Morphol., 28(4):1181-1188, 2010.

SUMMARY: Facial reconstruction for forensic sculpture is a procedure used in human identification. It consists of placing a mouldable material on the bone, respecting the soft tissue and facial anatomy. One of the main technical difficulties is the reproduction of structures of variable position as the eyes, ears and nose. It has been reported that the nose can be reconstructed reliably if you have pronasal point, that's why we have developed various techniques for the location of this point from the cranial bones. The purpose of this study is to evaluate by mean lateral cephalometric radiography the accuracy of four methods widely used for the location of the pronasal point from skulls. 34 lateral cephalometric radiography with soft-tissue facial profile taken under standardized conditions were used, on which tracings were made in hard and soft profile (control profile). Gerasimov, Krogman, Prokopec \& Ubelaker and George methods were evaluated, determining the differences in a coordinate axis $(\mathrm{X}, \mathrm{Y})$ with respect to the gold standard (pronasal point given in the soft profile). All methods had low levels of accuracy, underestimating the anterior projection of the nose. According to the averages, the method of Prokopec \& Ubelaker was the closest to the gold standard, with averages of 5.6 (SD 3.66) in X and 1.7 (SD 3.3 ) in Y, and higher levels of accuracy. The other methods tested were less accurate and had a high dispersion. The use of reliables and applicables methods for determining the pronasal point to our population are essential for the proper reconstruction of the nose.

KEY WORDS: Pronasal point; Forensic reconstruction; Human identification. 


\section{REFERENCIAS BIBLIOGRÁFICAS}

Enlow, D. H \& Hans M. G. Essentials of Facial Growth. Philadelphia: W. B. Saunders, 1996.

Gatliff, B. P. Facial sculpture on the skull for identification. Am. J. Forensic Med. Pathol., 5:327-32, 1984.

George, R. M. The lateral craniographic method of facial reconstruction. J. Forensic Sci., 32:1305-30, 1987.

George, R. M. Anatomical and artistic guidelines for forensic facial reconstruction. In: Iscan, M.Y.; Helmer, R. P, (editors). Forensic Analysis of the Skull. New York, WileyLiss, 1993. pp. 215-27.

Gerasimov, M. M. The Face Finder. New York, CRC Press, 1971.

Goldhamer, K. Rontgenologische Studien u" ber das menschliche Profil. I. Auszere Nasi. Z. Anatom. Entwickl., 81:115-50, 1926.

Krogman, W. M. \& Iscan, M. Y. The Human Skeleton in Forensic Medicine. $2^{\text {nd }}$ Ed. Springfield, IL, C.C. Thomas Publishers, 1986.

Larsen, W. J. Human Embryology. $3^{\text {rd }}$ Ed. Oxford, Churchill Livingstone, 2001.

Macho, G. A. An appraisal of plastic reconstruction of the external nose. J. Forensic Sci., 31:1391-1403, 1986.

Prag, J.; Neave, R. A. H. Making Faces. London, British Museum Press, 1997.

Prokopec, P \& Ubelaker, D. H. Reconstucting the shape of the nose according to the skull. Forensic Sci. Commun., 4:1, 2002.

Sobotta, J. Atlas of Human Anatomy. $10^{\text {th }}$ Ed. Ferner, H.; Staubesand, J. (editors). Baltimore, Urban and Schwazenberg, 1983. V.1.

Stephan, C.N.; Henneberg, M.; Sampson, W. Predicting nose projection and pronasale position in facial approximation: a test of published methods and proposal of new guidelines. Am. J. Phys. Anthropol., 122:240-50, 2003.

Stephan, C. \& Henneberg, M. Building faces from dry skulls: are they recognized above chance rates? J. Forensic Sci., 46:432-40, 2001.

Suk, V. Fallacies of anthropological dentifications and reconstructions: a critique based on anatomical dissections. Brno: Faculty of Science, University of Masaryk, 1935. PP. 1-18.

Suazo, G. I. C.; Cantín, L. M.; Zavando, M. D. A.; Perez, R. F. J. \& Torres, M. S. R. Comparisons in soft-tissue thickness on the human face in fresh and embalmed corpses using needle puncture method. Int. J. Morphol., 26(1):165-9, 2008 a.

Suazo, G. I. C.; Trujillo, H. E. G.; Cantín, L. M. G. \& Zavando, M. D. A. Determinación de proporciones áureas craneofaciales para la reconstrucción con fines de identificación medicolegal. Int. J. Morph., 26 (2):331-5, 2008 b.

Suazo, G. I. C.; Zavando, M. D. A \& Smith, R. L. Evaluating accuracy and precision in morphologic traits for sexual dimorphism in malnutrition human skull: a comparative study. Int. J. Morphol., 26(4):876-83, 2008c.

Suazo, G. I. C.; Zavando, M. D. A. \& Smith, R. L. Sex determination using mastoid process measurements in brazilian skulls. Int. J. Morphol., 26(4):941-4, 2008 d.

Suazo, G. I.; Zavando, M. D.; Smith, R. L. Performance evaluation as a diagnostic test for traditional methods for forensic identification of Sex. Int. J. Morphol., 27(2): 3816, 2009.

Trujillo, H. E. G.; Suazo, G. I. C., Cantín, L. M. \& Vargas, A. R. A. Determinación de nuevas proporciones áuricas craneofaciales. Int. J. Odontostomat., 1(2):165- 8, 2007.

Troncoso, P. J. A.; Suazo, G. I. C., Cantín L. M. \& Zavando. M. D. A. Sexual dimorphism in the nose morphotype in adult Chilean. Int. J. Morphol., 26(3):537-42, 2008.

Virchow, H. Die anthropologische untersuchung der nase. $Z$. Ethnol., 44:290-337, 1912.

Virchow, H. Zur Anthropologie der Nase. Z. Ethnol., 56:94$121,1924$.

Wilkinson, C. M. Forensic Facial Reconstruction. Cambridge, Cambridge University Press, 2004.

Dirección para Correspondencia

Prof. Dr. Iván Suazo Galdames

Departamento de Ciencias Básicas Biomédicas

Facultad de Ciencias de la Salud

Universidad de Talca

Email:isuazo@utalca.cl

Recibido : 27-08-2010

Aceptado: 26-09-2010 